\title{
Impact of Synchronous Condensers on Transmission Line Protection in Scenarios with High Penetration of Renewable Energy Sources
}

\author{
Dimitrios Tzelepis $^{\dagger}$, Eleni Tsotsopoulou ${ }^{\dagger}$, Vasileios Nikolaidis ${ }^{\star}$, Adam Dyśko ${ }^{\dagger}$ \\ Vasileios Papaspiliotopoulos ${ }^{\perp}$, Qiteng Hong ${ }^{\dagger}$, Campbell Booth ${ }^{\dagger}$ \\ ${ }^{\dagger}$ Department of Electronic \& Electrical Engineering, University of Strathclyde, Glasgow, UK \\ dimitrios.tzelepis@strath.ac.uk,a.dysko@strath.ac.uk,q.hong@strath.ac.uk campbell.d.booth@strath.ac.uk \\ * Department of Computer \& Electrical Engineering, Democritus University of Thrace, Greece \\ vnikolai@ee.duth.gr \\ $\perp$ PROTASIS Engineering \& Consulting, Athens, Greece \\ vpapaspiliotopoulos@protasis.net.gr
}

Keywords-Synchronous Condensers, Distance Protection, Renewable Energy Sources

\begin{abstract}
The objective of the studies presented in this paper aims to demonstrate that the deployment and operational control of synchronous condensers in the GB transmission system can mitigate a part of the challenges associated with the high penetration of renewable energy sources. These include the decline of short circuit level and the subsequent impact on transmission line protection schemes. The case studies include scenarios such as transmission-level faults, fault level calculation and assessment of distance protection performance. The results and observations included in the paper aim to highlight the means and supporting evidence for the benefits of synchronous condensers in the view of a fully de-carbonised power system.
\end{abstract}

\section{Introduction}

Traditional power systems are gradually evolving towards power networks with high penetration of renewable, inverterconnected generation (ICG). From the power generation perspective, centralised conventional synchronous generation plants are gradually replaced by renewable generation, connected both to transmission and distribution level.

This change in generation mix creates significant system issues which may lead to certain risks that need to be managed. One of the most important challenges is associated with the decline of short circuit levels (SCLs) which can result in poor power quality and also lead in certain protection schemes (i.e. distance and over-current protection) failing to operate. On certain circumstances, low SCL can escalate the risk of a cascading tripping event (and the potential for system blackout) as a result of the tripping of anti-islanding protection (e.g. RoCoF) applied to distributed generation, which would clearly exacerbate the initial system disturbance [1], [2].

In order to mitigate the aforementioned challenges, synchronous condenser (SynCon) solutions have been recently considered by transmission system operators in UK [3], Denmark [4] and California [5], as a mean to support transmission systems by offering short circuit current, dynamic reactive power provision and system inertia.
The studies presented in this paper will aim to demonstrate that the deployment and operational control of SynCon units in the GB transmission system, can mitigate a part of the challenges associated with the high penetration of renewable energy sources focusing on short circuit levels and distance protection performance.

\section{Modelling}

The simulation-based analysis and the corresponding results presented in this paper will consider both static and transient simulation environments.

\subsection{Static/RMS model}

The studies conducted through this simulation environment are based on a model of national electricity transmission system of GB (which is represented by a 36-bus equivalent network) and will aim to assess the fault level at GB transmission system at various regions. The model has been developed by NationalgridESO in DIgSILENT PowerFactory and the network topology is depicted in Figure 1.

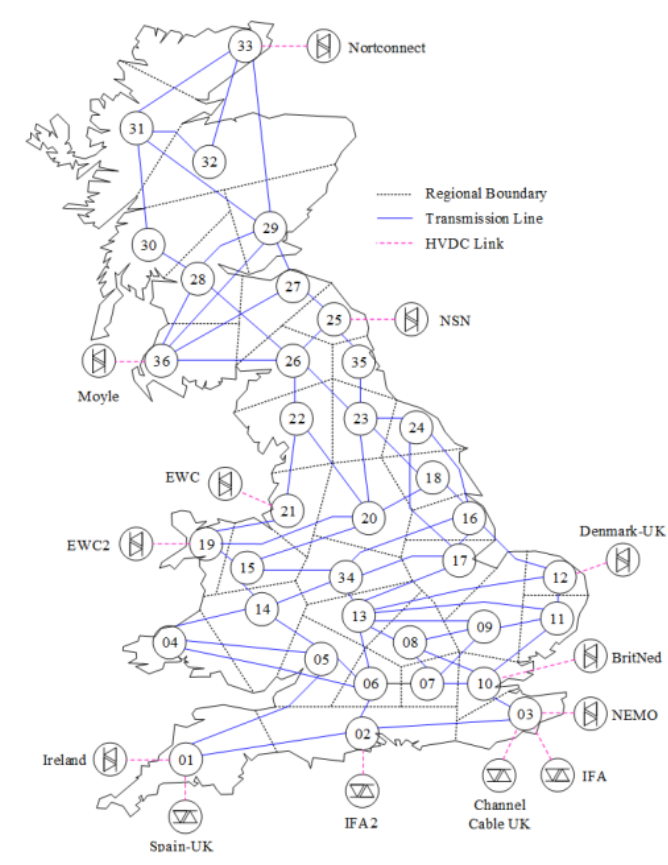

Figure 1: Transmission network of GB: 36-zone equivalent. 
Each numbered node in the model represents a part of the system and consists of a mix of different energy sources and loads. Generators within each zone are represented by synchronous machines (including relevant dynamic controllers) and static generators (to represent ICG). At each zone, HVDC interconnectors, loads, generation units and transmission lines are connected to $400-\mathrm{kV}$ busbars.

The fault level calculations will utilise the superposition method for the SCL calculation and will report the indices of peak short-circuit current $I_{p}$ (instantaneous value) and shortcircuit power $S_{k}$. The studies will be repeated for different SynCon capacities, location (zone-wise) and generation setup.

\subsection{Transient model}

The studies conducted through this simulation environment will aim to assess the performance of distance protection relays in the occurrences of transmission line faults. For the purposes of carrying out transient simulation analysis, a part of a typical transmission network model has been developed in Matlab/Simulink (the network topology is shown in Figure 2).

The network operates at $400 \mathrm{kV}$ and consists of a 100$\mathrm{km}$ transmission line, a grid (represented by a voltage source) and three different generation units accounting for synchronous generator (SG), SynCon and a wind farm connected via a voltage source converter (VSC).

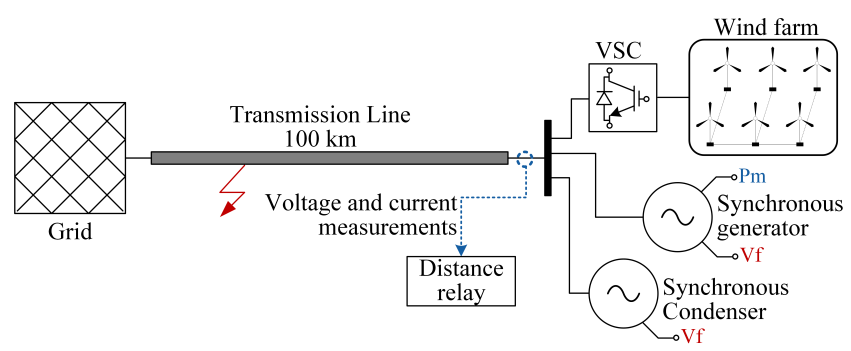

Figure 2: Transmission network illustrating integration of SGs, SynCons and ICG.

The SG and SynCon units are modelled as a standard salientpole synchronous machine. Three control systems have been integrated which include i) automatic voltage regulator, ii) power system stabiliser and iii) over excitation limiter. In the case of SynCon unit, the prime mover and governor have been omitted as well (i.e. there is no mechanical input $P_{m}$ ). Wind farms constitute of permanent magnet synchronous generators connected via VSCs, which operate under the standard D-Q axis current injection (DQCI) control scheme.

A validated dynamic model of a commercially available distance protection relay (i.e. 'MICROMHO - Static Distance Protection Relay') has been utilised in this analysis. Timedomain voltage and current signatures are captured from the generation terminals and imported to the relay for post processing. The studies consider scenarios under different generation mixes starting from $100 \%$ synchronous to $100 \%$ ICG. In the latter setup, SynCon units under different capacities are deployed to qualify and quantify their impact on transmission line protection in terms of number of successful trips and response time.

\section{Simulation Results}

\subsection{Short Circuit Level}

For the calculation of SCL and the quantification of the contribution of SynCon units, it is of major importance to define and understand the method for calculating the SCL; the results reported here deploy the superposition method. In this method, the pre-fault load conditions of the network are considered (the load flow and the setting of the transformer tap changers are a pre-requisite) and the calculations are executed in three discrete stages as depicted in Figure 3.

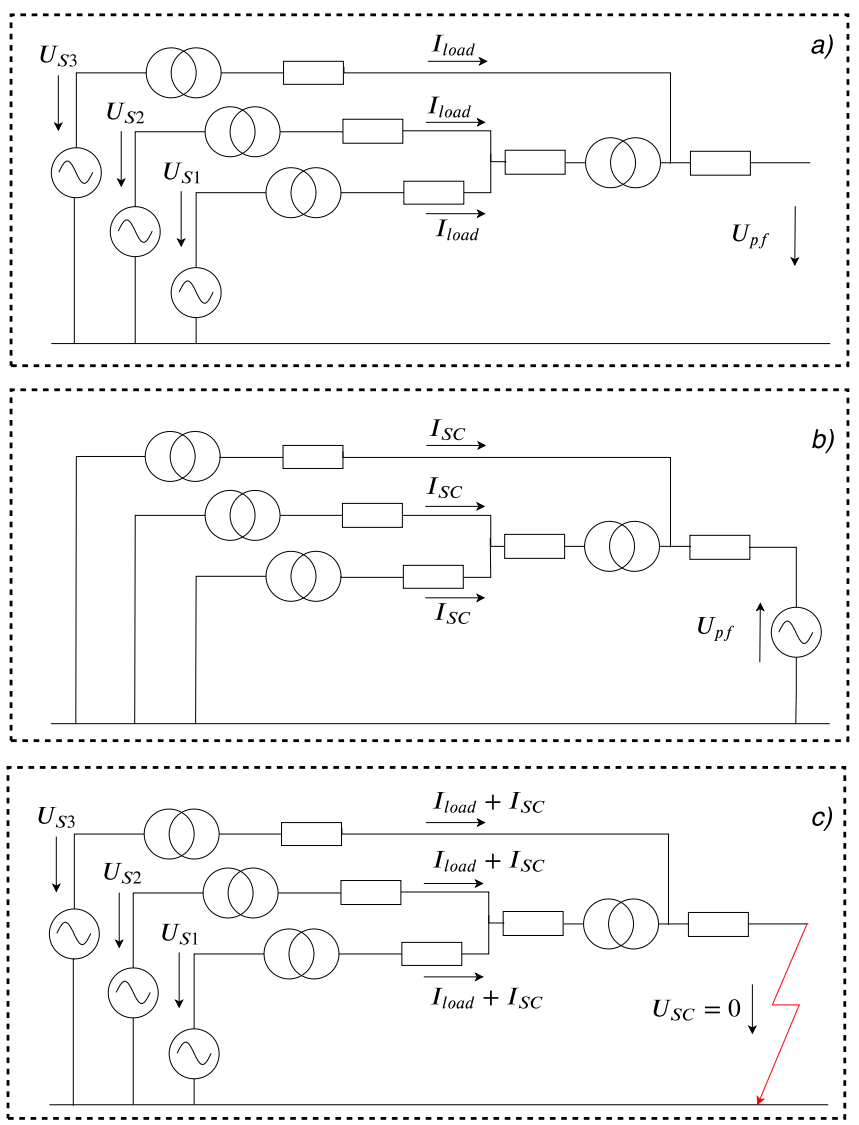

Figure 3: Stages of SCL calculation using super-position method: a) pre-fault, b) pure-fault, c) post-fault.

It should be noted that since SynCon units are the main focus of this analysis, their representation though the superposition method can be considered as voltage sources behind an impedance. The stages for the SCL calculation are analysed as follows:

- Pre-fault: The calculation begins with the definition of the normal operating conditions (refer to Figure 3a). These conditions reflect the tap changer position of transformers, load conditions, breakers status and excitation of generators. At this stage the pre-fault voltage $U_{p f}$ at the fault position is determined.

- Pure-fault: At the second stage (refer to Figure 3b), a negative version of pre-fault $U_{p f}$ is applied to the fault position and all the remaining sources are set to zero. Essentially, $U_{p f}$ is the only voltage source in the network under study.

- Post-fault: The system performance after fault inception (refer to Figure 3c) is determined by 
complex-adding both the pre-fault and pure-fault conditions.

Prior to the quantification of SynCon contribution, the SCL (accounting for short-circuit power $S_{k}$ and shortcircuit peak current $I_{p}$ ) has been calculated (without the contribution of SynCon units) for the different dispatch scenarios reflecting years 2019 and 2023 (both winter peak and summer minimum periods) of UK generation and demand background. The results are illustrated in Figure $4 \mathrm{a}$ and Figure $4 \mathrm{~b}$, for $S_{k}$ and $I_{p}$ respectively.
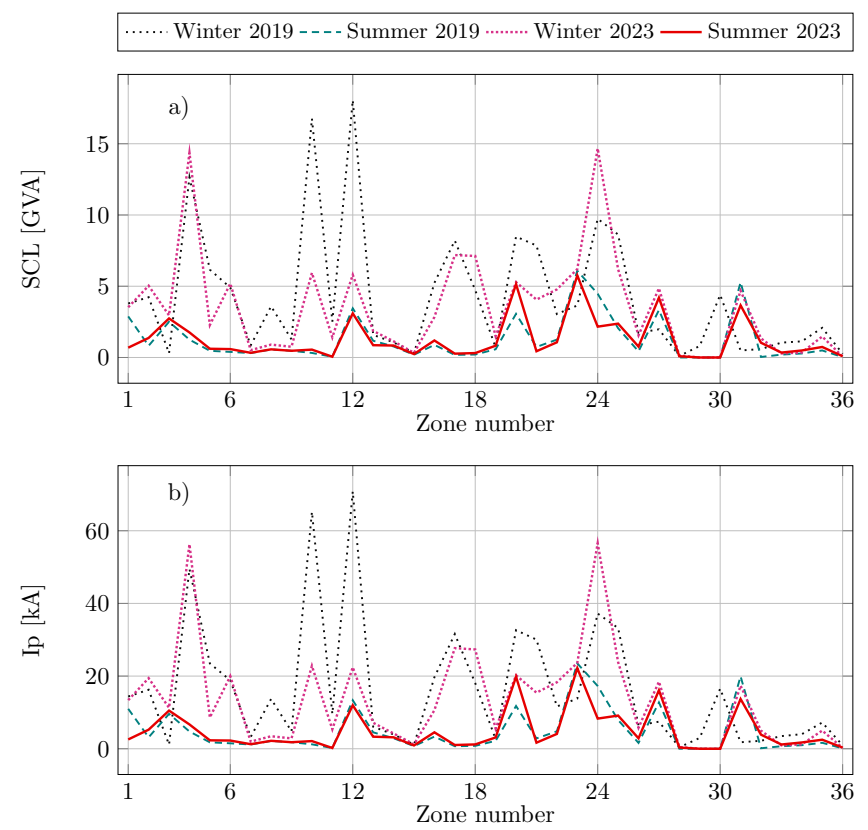

Figure 4: SCL results at different zones and for different SynCon capacity: a) short-circuit power $S_{k}$, b) short-circuit peak current $I_{p}$.

Figure 4 highlights that there is a decline in SCL especially during summer periods. Such a decline can result in poor power quality and also lead in certain protection schemes (i.e. distance and over-current protection) failing to operate. Therefore this calls for systematic evaluation and investigation for solutions enabling SCL elevation such as SynCon units as proposed in this paper.

In order to calculate the contribution of SynCon units to the SCL, a set of simulation scenarios has been setup to incrementally change the capacity of SynCon units at each zone. The maximum capacity has been set to 700 MVA with increments of 70 MVA. The short-circuit power $S_{k}$ and shortcircuit peak current $I_{p}$ have been captured. To minimise the number of simulations, for each dispatch scenario, the zones entailing the minimum SCL has been identified and selected as a benchmark case. These correspond to zones 28, 30, 30 and 30 for dispatch scenarios Winter 2019, Summer 2019, Winter 2023 and Summer 2023 respectively.

The results for the contribution of SynCon units to the SCL are depicted in Figure 5 and Figure 6, for $S_{k}$ and $I_{p}$ respectively. From those figures it can be seen that the addition of SynCon units can definitely increase both shortcircuit power $S k$ and short-circuit peak current $I_{p}$. The presented trends suggest that the amount of SCL elevation is approximately four times the rated power of the machine, which is well-aligned with the conventional fault analysis theory. Additionally, the results of such an analysis, can be taken into consideration when conducting studies relevant to protection settings and coordination [6], [7].
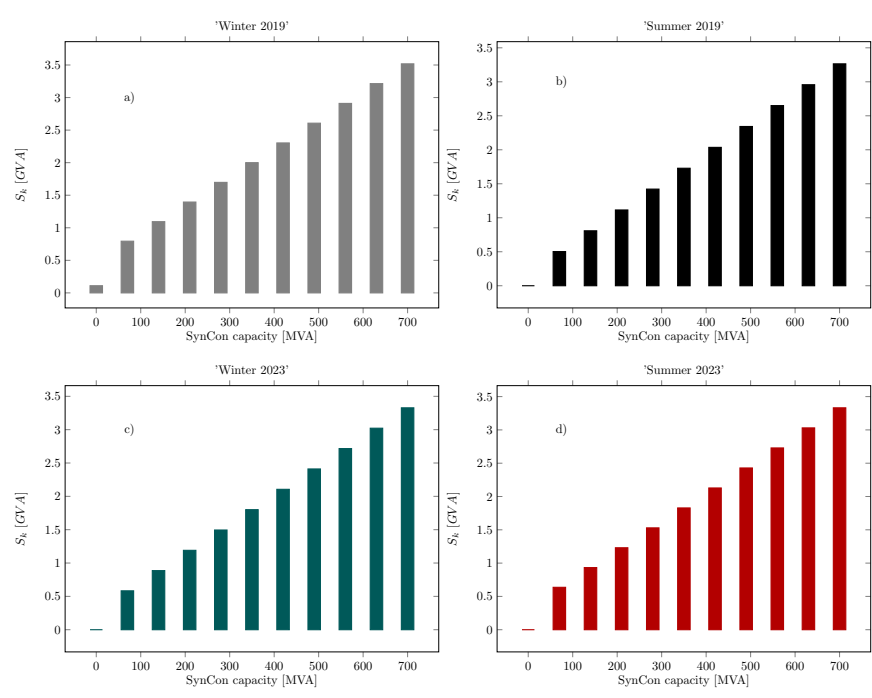

Figure 5: SCL results accounting for short-circuit power $S_{k}$ for different SynCon capacities: a) Zone 28 - Winter 2019, b) Zone 30 - Summer 2019, c) Zone 30 - Winter 2023, d) Zone 30 - Summer 2023.
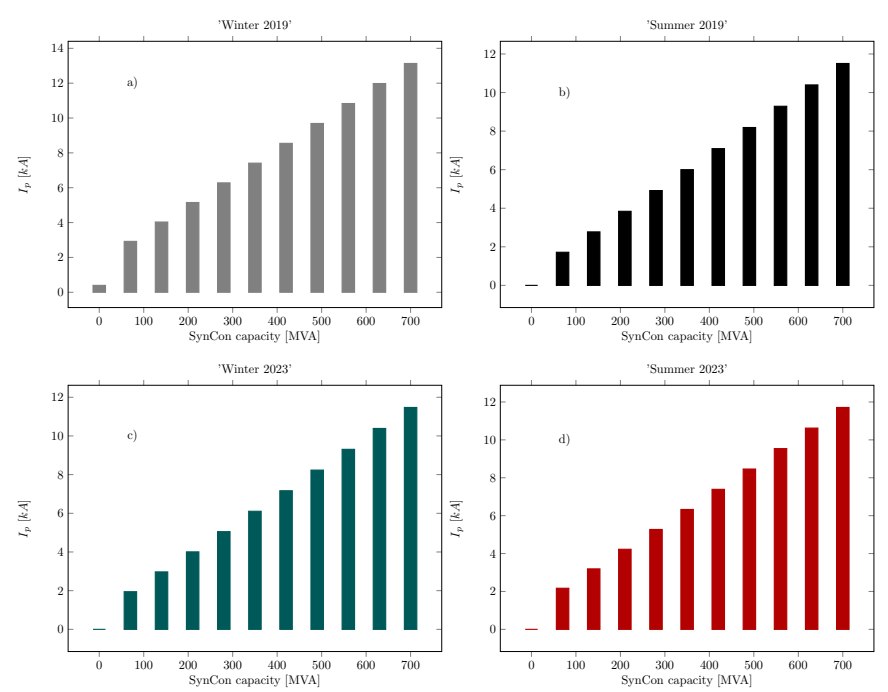

Figure 6: SCL results accounting for short-circuit peak current $I_{p}$ for different SynCon capacities: a) Zone 28 Winter 2019, b) Zone 30 - Summer 2019, c) Zone 30 - Winter 2023, d) Zone 30 - Summer 2023.

\subsection{Distance protection performance}

Prior to the quantification of the contribution of SynCon units to the performance of distance protection, it is of utmost importance to demonstrate the challenges related to fault currents when ICG reaches high levels of penetration.

Figure 7 demonstrates the fault current signatures during three-phase transmission line faults for different generation mixes. When the generation is solely based on SG (refer to Figure 7a), fault current reaches instantaneous values up to 
$12 \mathrm{pu}$. On the contrary, when there is $100 \%$ of ICG (refer to Figure $7 \mathrm{~b}$ ), fault current contribution is limited to $1.2 \mathrm{pu}$. This emanates from the fact that control schemes of the inverters (especially DQCI schemes) limit and balance their output to protect the power electronic valves from overheating. In the presence of $100 \%$ ICG, when SynCon units are connected, the fault current contribution can be enhanced. Figure $7 \mathrm{c}$ illustrates the fault current contribution when $20 \%$ of SynCon capacity is added at the generation mix (please note that this additional $20 \%$ does not count as generation, as there is no active power delivered by the SynCon unit). It is evident that the values of current can be elevated and the performance of the protection schemes is anticipated to be improved.
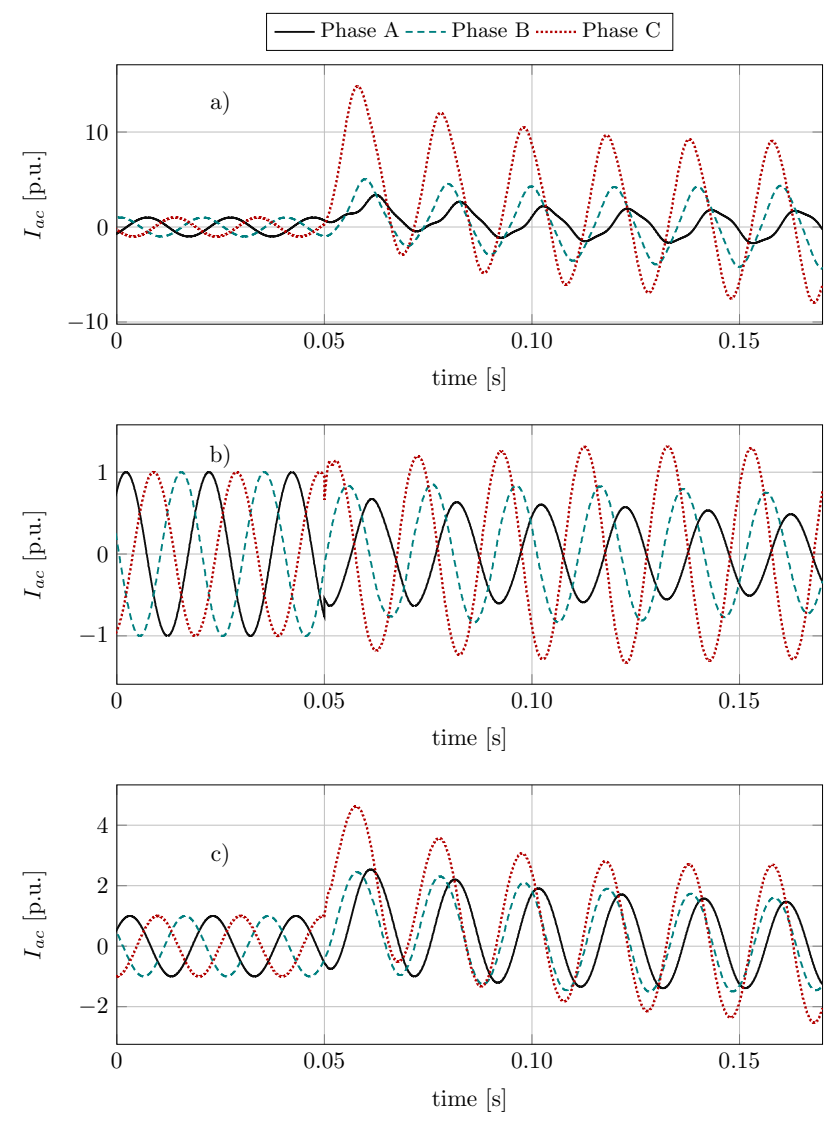

Figure 7: Fault current signatures for three-phase transmission line faults for different generation mixes: a) $100 \% \mathrm{SG}$, b) $100 \% \mathrm{ICG}$, c) $100 \%$ ICG + $20 \%$ SynCon.

The fault current signatures shown in Figure 7 have been imported to the relay (alongside with the corresponding voltage traces). In Figure 8, the characteristics of the mho impedance relay are plotted on a R-X locus. The results verify that the operation of the distance relay depends on the penetration level of the non-synchronous generation and the utilisation of SynCon can improve the reliability of distance protection.

To quantify the contribution of SynCon to the performance of distance protection, a series of simulation test-sets have been conducted for different generation scenarios, fault resistance values, fault positions along the line and fault types. The analysis considered three types of faults within Zone 1 of the protected line: three-phase, phase-phase and singlephase to ground with fault resistances up to $150 \Omega$. In total, the distance protection relay has been tested under 2,223

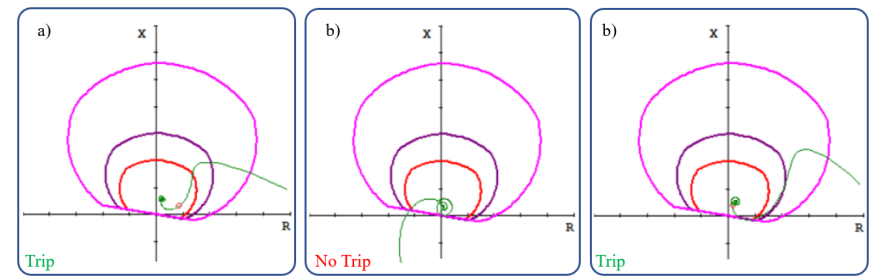

Figure 8: Distance protection R-X locus for three-phase transmission line faults for different generation mixes: a) $100 \%$ SG (relay tripped), b) $100 \%$ ICG (relay did not trip), c) $100 \%$ ICG $+20 \%$ SynCon (relay tripped).

\section{different faults scenarios.}

The generation scenarios for this analysis are presented in Table 1, where three dominant generation technologies are included (i.e. SG, ICG and SynCon). These groups subsequently formed nine distinct generation mixes including single technology connections, as well as mixes of two (the base power has been set to $S_{b a s e}=500 M V A$ ). For all the scenarios an automatic search routine has been developed to iteratively change the position of the fault along the line, fault resistance and fault type.

TABLE 1: Portion of generation technologies within a 500 MVA generation mix.

\begin{tabular}{cccc}
\hline \hline Scenario & SG & ICG & SynCon \\
\hline 1 & $S_{b}$ & - & - \\
2 & $0.75 \cdot S_{b}$ & $0.25 \cdot S_{b}$ & - \\
3 & $0.50 \cdot S_{b}$ & $0.5 \cdot S_{b}$ & - \\
4 & $0.25 \cdot S_{b}$ & $0.75 \cdot S_{b}$ & - \\
5 & - & $S_{b}$ & - \\
6 & - & $S_{b}$ & $0.05 \cdot S_{b}$ \\
7 & - & $S_{b}$ & $0.10 \cdot S_{b}$ \\
8 & - & $S_{b}$ & $0.15 \cdot S_{b}$ \\
9 & - & $S_{b}$ & $0.20 \cdot S_{b}$ \\
\hline \hline
\end{tabular}

Figure 9 depicts the number of successful trips for each of the generation scenario. The percentage of the successful operations has been calculated by dividing the total number of the correct operations by the number of the desired trips for each scenario. The number of the desired trips is based on the definition of the power protection dependability, which is the degree of ability of the protection system to operate correctly for faults within the protected zone.

It can be seen that as the proportion of ICG increases, the operation of the relay is compromised. This behaviour is more pronounced at Scenario 5 (i.e. 100\% ICG) where the number of successful trips reaches only $20 \%$. However, when SynCon is deployed in the system (i.e. scenarios 6 to 9), the percentage of successful relay trips increases, reaching values up to $60 \%$.

The impact of SynCon units has been quantified against the response time of the distance protection (response time is defined as the time lapsed between the fault occurrence and the time when the trip signal is initiated). The average response time for all the fault scenarios is depicted in Figure 10. It is evident that the as the penetration of ICG increases, the response time increases. Specifically, when the generation mix consists of $100 \% \mathrm{SG}$, the response time is approximately $20 \mathrm{~ms}$ (i.e. 1 electrical cycle) while for $100 \%$ ICG the response time reaches values approximately up to $70 \mathrm{~ms}$. When SynCon units are utilised, the average response time 


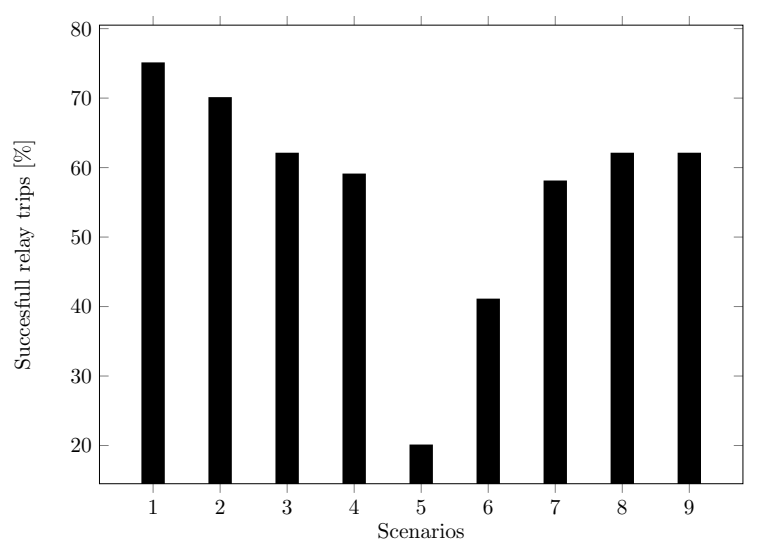

Figure 9: Number of successful trips.

starts improving reaching values close to $40 \mathrm{~ms}$, hence improving the speed of protection operation.

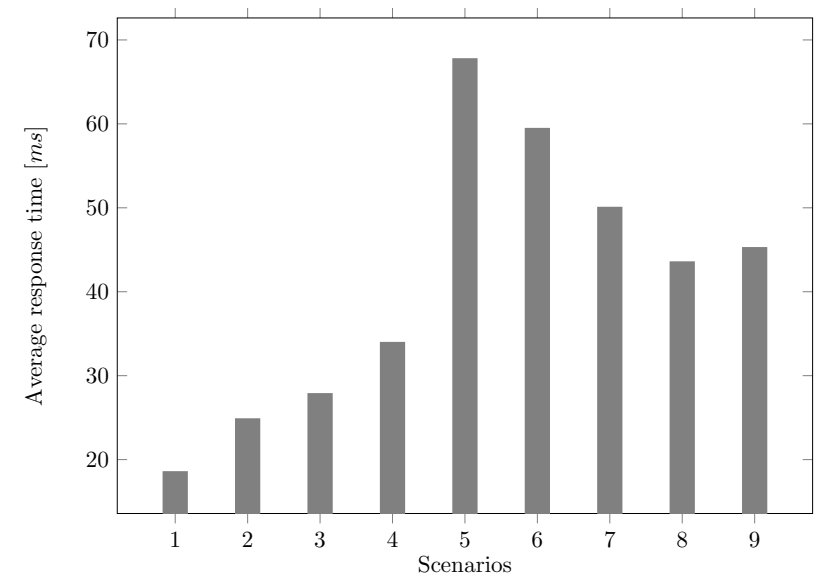

Figure 10: Average response time.

\section{Conclusions}

In this paper synchronous condenser units have been investigated to mitigate challenges associated with the high penetration of renewable energy sources with emphasis given to short circuit level and the performance of distance protection schemes. The simulation-based analysis and the corresponding results presented in this paper considered both static and transient simulation environments. The utilisation of synchronous condenser units in the GB transmission system has been found to be beneficial for the system by increasing the short circuit level accounting for shortcircuit power and short-circuit peak current. It has been demonstrated that when the penetration of inverter-connected generation reaches $100 \%$, the performance of distance protection is compromised (accounting for detection and response time) due to the limited fault current. At this case, it has been revealed that the deployment of synchronous condenser units can increase the number of successful trips and also decrease the response time of distance relays, hence improving the dependability and sensitivity of distance protection schemes.

\section{Acknowledgements}

This research is supported by SP Energy Networks and NationalGridESO Grid under the PHOENIX project (reference: SPTEN03/Phoenix).

\section{References}

[1] A. Dyśko, D. Tzelepis, C. Booth, J. Pollock, and D. Hill, "Practical risk assessment of the relaxation of lom protection settings in NIE networks' distribution system," The Journal of Engineering, vol. 2018, no. 15, pp. 1335-1339, 2018.

[2] D. Tzelepis, A. Dyśko, and C. Booth, "Performance of loss-of-mains detection in multi-generator power islands," in 13th International Conference on Development in Power System Protection (DPSP), March 2016, pp. 1-6.

[3] D. Tzelepis, Q. Hong, C. Booth, P. Papadopoulos, J. Ramachandran, and G. Yang, "Enhancing short-circuit level and dynamic reactive power exchange in GB transmission networks under low inertia scenarios," in International Conference on Smart Energy Systems and Technologies, Porto, Portugal, September 2019.

[4] J. Jia, G. Yang, A. H. Nielsen, and V. Gevorgian, "Investigation on the combined effect of VSC-based sources and synchronous condensers under grid unbalanced faults," IEEE Transactions on Power Delivery, pp. 1-1, 2019.

[5] G. Zhou, D. Wang, A. Atallah, F. McElvain, R. Nath, J. Jontry, C. Bolton, H. Lin, and A. Haselbauer, "Synchronous condenser applications: Under significant resource portfolio changes," IEEE Power and Energy Magazine, vol. 17, no. 4, pp. 35-46, July 2019.

[6] M. N. Alam, "Adaptive protection coordination scheme using numerical directional overcurrent relays," IEEE Transactions on Industrial Informatics, vol. 15, no. 1, pp. 64-73, Jan 2019.

[7] V. A. Papaspiliotopoulos, G. N. Korres, and N. G. Maratos, "A novel quadratically constrained quadratic programming method for optimal coordination of directional overcurrent relays," IEEE Transactions on Power Delivery, vol. 32, no. 1, pp. 3-10, Feb 2017. 